UDC 616.24: 578.834.1]: 519.24

DOI: 10.21668/health.risk/2021.1.03.eng

Research article

\title{
SPATIO-TEMPORAL MODELING OF COVID-19 EPIDEMIC
}

\author{
V.L. Sokolovsky ${ }^{1}$, G.B. Furman ${ }^{1}$, D.A. Polyanskaya ${ }^{2}$, E.G. Furman ${ }^{2}$ \\ ${ }^{1}$ Ben Gurion University of the Negev, P.O.B. 653, Beer-Sheva, 84105, Israel \\ ${ }^{2}$ Perm State Medical University named after Academician E.A. Wagner, 26 Petropavlovskaya Str., Perm, 614000, \\ Russian Federation
}

In autumn and winter 2020-2021 there was a growth in morbidity with COVID-19. Since there are no efficient medications and mass vaccination has only just begun, quarantine, limitations on travels and contacts between people as well as use of personal protection equipment (masks) still remain priority measures aimed at preventing the disease from spreading.

In this work we have analyzed how the epidemic developed and what impacts quarantine measures exerted on the disease spread; to do that we applied various mathematical models. It was shown that simple models belonging to SIR-type (S means susceptible; I, infected; and $R$, recovered or removed from the infected group) allowed estimating certain model parameters such as morbidity and recovery coefficients that could be used in more complicated models.

We examined spatio-temporal epidemiologic models based on finding solutions to non-stationary two-dimensional reaction-diffusion equations. Such models allow taking into account uneven distribution of population, changes in population mobility, and changes in frequency of contacts between susceptible and infected people due to quarantine. We applied obtained analytical and numerical solutions to analyze different stages in the epidemic as well as its wave-like development influenced by imposing and canceling quarantine limitations.

To take into account ultimate rate at which the disease spreads and its incubation period (when an infected person is not a source of contagion), we suggest using equations similar to the Cattaneo-Vernotte one. The suggested model allows predicting where the front of morbidity spread is going to occur, that is, a moving frontier between areas where there are infected people and areas where they are absent. Use of such models provides an opportunity to introduce differential quarantine measures basing on more objective grounds.

At the end of 2020 mass vaccination started in some countries. We estimated a necessary number of people that had to be vaccinated so that new waves of COVID-19 epidemic would be prevented; in our estimates, not less than $80 \%$ of the country population should be vaccinated.

Correct prediction of epidemic development is becoming more and more vital at the moment due to new and more contagious COVID-19 virus strains occurring in England, South Africa, and some other countries.

Our research results can be used for predicting spread of COVID-19 and other communicable diseases; they can make for taking the most efficient measures for successful control over epidemics.

Key words: spatio-temporal modeling, epidemic, COVID-19, morbidity, non-stationary two-dimensional reactiondiffusion equations, quarantine, contagion rate, recovery rate.

Researchers predict that morbidity with COVID-19 will grow in winter 2020-2021 [1]. In autumn 2020 there is an intense growth in number of people infected with COVID-19 all over the world and it is the second pandemic wave. Lockdown is again introduced in some European countries and RF regions as well. In Israel there was a repeated rapid growth in morbidity in late August and September, and it was only strict lockdown introduced at the end of September and was valid for the whole October that allowed reducing quantity of everyday contagion cases and seriously ill patients as well as patients who needed to be switched to artificial lung ventilation devices.

(C) Sokolovsky V.L., Furman G.B., Polyanskaya D.A., Furman E.G., 2021

Vladimir L. Sokolovsky - Candidate of Sciences, Professor at the Physics Department (e-mail: sokolovv@bgu.ac.il; tel.: +9 (725) 464-703-40; ORCID: https://orcid.org/0000-0003-4887-413X).

Grigoriy B. Furman - Candidate of Sciences, Professor at the Physics Department (e-mail: gregoryf@bgu.ac.il; tel.: +9 (725) 476-842-45; ORCID: https://orcid.org/0000-0001-7303-9414).

Darya A. Polyanskaya - Candidate of Medical Sciences, Lecturer at the Hygiene Department of the Medical and Prevention Faculty (e-mail: daryasp88@gmail.com; tel.: +7 (909) 731-11-37; ORCID: https://orcid.org/0000-0002-1466-7039).

Evgeniy G. Furman - Corresponding member of the RAS, Doctor of Medical Sciences, Professor, Head of the Department for Faculty and Hospital Pediatrics (e-mail: furman1@yandex.ru; tel.: +7 (912) 883-97-35; ORCID: https://orcid.org/0000-0002-1751-5532). 
Since there are no efficient medications and vaccines against COVID-19, basic means that can prevent the disease spread are still lockdowns, limitations on travelling and contacts between people, as well as personal protection means such as facial masks. On one hand, lockdown introduction leads to a decrease in number of sick people and burden on a public healthcare system. On the other hand, lockdown results in a fall in economic activities, especially regarding trade, tourism, culture, and entertainment; it also results in deteriorating quality of education and does certain damage to mental state of population. Lockdown should be introduced basing on analyzing how a disease spreads and mathematical models that take into account specific peculiarities of a given disease and are able to predict its spread. Developed procedures for mathematical modeling are significant not only for predicting COVID-19 spread and making for the most efficient measures being taken but also for successful fighting against future epidemics.

Various mathematical models showing epidemic development were developed and applied when analyzing variable diseases spread (typhus, cholera, Ebola, etc.) [2-7]. These models, as well as some new ones that took into account COVID-19 peculiarities, were used for simulating COVID-19 epidemic [8-15]. Starting from the very beginning of COVID-19 epidemic, more than 11 thousand articles have been published in scientific journals and on specialized web-sites, as well as in medRxiv, bioRxiv and arXiv archives; these works focus on various aspects of the disease including statistics and analysis of its spread. Most models are based on the SIR ( $S$ - susceptible, $I$ - infected, $R$ - removed from infected or recovered) epidemic model and its modifications.

These models are based on an assumption that susceptible, infected, and recovered people are spatially distributed evenly. But in actual conditions these population groups are distributed extremely unevenly even in small countries and megacities. There are also several models that take into account uneven spatial distribution of susceptible and infected people [2, 4, 7]. Reaction-diffusion epidemi- ologic models belong to such models with a disease spread being given in them with a system of heat conductivity equations with chemical reaction. However, these models do not take into account natural daily people migration (home, work, educational establishments, cultural institutions, etc.). Another drawback is an assumption that virus is caught from infected people instantly and it simultaneously moves people from susceptible into infected ones and as a result a disease spreads with infinite rate.

Our research goal is to analyze spread of COVID-19 epidemic using various epidemiologic mathematical models. Spatio-temporal epidemic modeling is based on analytical and numerical solutions to non-stationary twodimensional reaction-diffusion equations as well as these equations that model a time lag in a disease occurrence.

Data and methods. In the $1^{\text {st }}$ section of Results and discussion, we used the SIR model and statistical data to determine morbidity and recovery coefficients; then, introducing dependences of these coefficients on time, we modeled a wave-like epidemic caused by lockdown being introduced and canceled. In the next section we showed that morbidity and recovery coefficients determined with the SIR model could be used in reaction-diffusion epidemiologic models; we also analyzed twodimensional spread of the disease. In the $3^{\text {rd }}$ section we modernized reaction-diffusion epidemiologic models and it allowed us to model spatio-temporal epidemic development taking into account daily population migration. In the $4^{\text {th }}$ section we introduced a reaction-diffusion epidemiologic model with finite contagion time. The suggested model predicts that a front of the disease spread will occur, that is, a moving frontier between areas where there are infected people and areas where they are absent.

Results and discussion. 1. SIR epidemic model. In this section we consider the simplest SIR model that allows assessing COVID-19 epidemic dynamics in the $1^{\text {st }}$ approximation and is a basis for developing more precise models. Within the SIR epidemic model population is divided into groups that do not overlap and number of people in them changes over time $[2-4,6,9]$. A «susceptible» group 
includes people who are prone to infection but who are not sick yet. The number of susceptible group $S(t)$ goes down due to people moving to an «infected» group: number of infected people $I(t)$ goes down due to people making full recovery or dying $(R(t))$. Recovered people are assumed to have immunity; transfer from one group to another does not depend on age, sex, social status, etc.

Basic assumptions that underlie use of this model are the following:

- each person from the susceptible group contacts infected ones and there is a certain probability that he or she will also get infected, that probability does not depend on time;

- infection rate is proportionate to a number of infected people as well as quantity of susceptible people;

- each infected person, in his or her turn, is constantly likely to recover at a certain time unit;

- recovery rate is proportionate to a number of infected people;

- a recovered person has immunity and is not susceptible to repeated infection.

SIR model equations can be given as:

$$
\begin{aligned}
& \frac{d s}{d t}=-\alpha_{1} s i \\
& \frac{d i}{d t}=\alpha_{1} s i-\alpha_{2} i \\
& \frac{d r}{d t}=\alpha_{2} i
\end{aligned}
$$

where $\quad s(t)=S(t) / N, \quad i(t)=I(t) / N$, $r(t)=R(t) / N, \quad \alpha_{1}$ and $\alpha_{2}$ are the morbidity and recovery coefficients respectively. These coefficients usually have dimension 1/day (1/d). Here it is assumed that population number

$$
S(t)+I(t)+R(t)=N
$$

is constant.

Numeric solution to the equations (1)-(3) at $\alpha_{1}=0.41 / \mathrm{d}, \alpha_{2}=0.11 / \mathrm{d}$ and initial conditions $s(0)=1, i_{0}=i(0)=10^{-6}$ and $r(0)=0$ is given in Figure 1.

$i(0)=10^{-6}$ for such countries as Israel, Greece, Sweden, as well as for megacities (Moscow, Saint-Petersburg) means that an epi- demic started with approximately 10 infected persons. Initial number of infected persons $i(0)$ (zero patients) does not influence the maximum number of infected $i_{m}$ and recovered people by the end of an epidemic $r_{l}=\left.r(t)\right|_{t \rightarrow \infty}$ (Figure 1). An epidemic will end up naturally when collective immunity is achieved and there are no new infected people. Variations in initial number of infected people lead to changes in a period of time during which an epidemic reaches its peak and then ends.

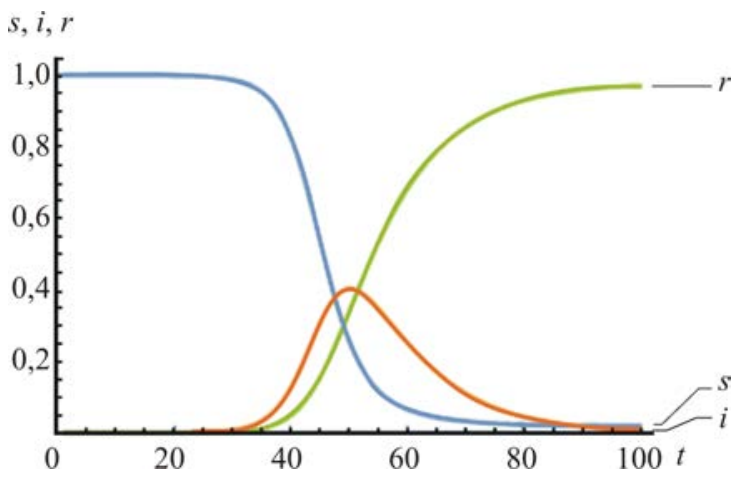

Figure 1. Solution to equations (1)-(3) at $R_{0}=4, \alpha_{1}=0.41 / \mathrm{d}$ and $\alpha_{2}=0.11 / \mathrm{d}$

Parameters $\alpha_{1}$ and $\alpha_{2}$ determine growth rates for a number of infected $i(t)$ and recovered $r(t)$ people. When an epidemic has just started and $i$, we can consider $s(t)=1$. On October 30, 2020 approximately 45.7 million people out of 7.8 billion world population all over the world got infected starting from the beginning of the epidemic; approximately $0.6 \%$ out of them (1.2 million) died (lethality is equal to $2.6 \%$ ). In Israel, approximately 314 thousand people out of 9.5 million are infected, that is, approximately $3 \%$, and 2,511 people died (lethality is $0.8 \%$ ). Despite COVID-19 epidemic has been lasting for several months already and there are huge numbers of people who got infected and died from COVID-19, approximation $s(t) \approx 1$ still holds quite satisfactorily. When performing mathematical modeling, we can also assume that all infected people recover.

At an initial stage in an epidemic a solution to the system of equations (1)-(3) is given as 


$$
\begin{gathered}
s(t) \approx 1 \\
i(t)=i_{0} e^{\alpha_{2}\left(R_{0}-1\right) t} \\
r(t)=\frac{1}{R_{0}-1}\left[e^{\alpha_{2}\left(R_{0}-1\right) t}-1\right]
\end{gathered}
$$

Basic reproduction number, that is, how many people are infected by a single infected person, $R_{0}=\frac{\alpha_{1}}{\alpha_{2}}$ is a significant parameter that determines a character of an epidemic, maximum number of infected $i_{m}$ and recovered people by the end of an epidemic $r_{l}$ (Figure 2) [10].

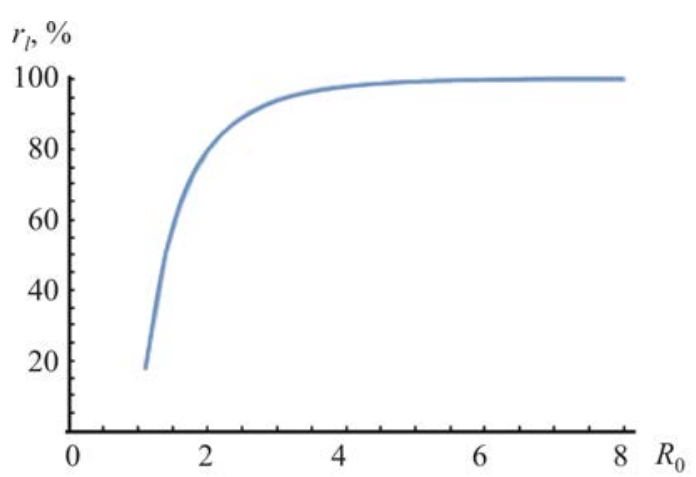

Figure 2. Number of recovered people by the end of an epidemic $r_{l}=\left.r(t)\right|_{t \rightarrow \infty}$ as a function of $R_{0}$ at $\alpha_{2}=0.11 / \mathrm{d}$ and initial conditions $s(0)=1$,

$$
i(0)=i_{0}=10^{-6} \text {, and } r(0)=0
$$

At $R_{0}<1$ a number of infected people goes down naturally without anti-epidemic measures being taken; an epidemic ends up.

At $R_{0}>1$ an epidemic will develop until collective immunity is formed. When basic reproduction number is low, $R_{0}=1.1$, collective immunity is formed when approximately $18 \%$ population overcame the disease (recovered people) at $\alpha_{1}=0.111 / \mathrm{d}, \alpha_{2}=0.11 / \mathrm{d}$ (Figure 2). This percent grows rapidly as $R_{0}$ increases and reaches $94 \%$ at $R_{0}=3$.

Computations have revealed that a share of infected (recovered) people that is necessary for collective immunity formation when a number of new infected people is about zero does not at all depend on parameters $\alpha_{1}$ and $\alpha_{2}$, and basically is determined by their ratio. However, a period of time necessary for reaching this immunity is inversely proportionate to these parameters: at $\alpha_{1}=0.31 / \mathrm{d}$ and $\alpha_{2}=0.11 / \mathrm{d}$ this period is estimated as being equal to 130 days; at $\alpha_{1}=0.031 / \mathrm{d}$ and $\alpha_{2}=0.01 \mathrm{1} / \mathrm{d}$, to 1,300 days.

The maximum number of infected people $i_{m}$ is also determined by a basic reproduction number $R_{0}$, and a period of time necessary to reach it starting from the beginning of an epidemic is determined by $\alpha_{1}$ and $\alpha_{2}$ parameters. The maximum number of infected people $i_{m}$ is reached at $s=\alpha_{2} / \alpha_{1}$ and overall number of infected were $N\left(1-1 / R_{0}\right)$. Further epidemic development is associated with a decrease in number of infected people though their number can reach greater values (Figure 1).

1.1. Model parameters assessment. Parameters that are components in SIR and other models cannot be measured directly since they depend on numerous factors, such as population density, population mobility, social contacts, lockdowns and how people adhere to quarantine measures, age structure of population, etc. $[8,9,10,16]$. Assessments are based on statistical data, their completeness and validity, as well as on establishing a clear correlation between them and «external» factors such as lockdown introduction, seasonal fluctuations, vacations and holidays, that determine authenticity of obtained estimations. Contagion intensity and, consequently, model parameters will depend on time [10, 16, 17]. Basic reproduction numbers $R_{0}$ given in literature depend not only on a country and time of analysis but also on an applied epidemiologic model [10, 17]. The authors of publication [11] estimated basic reproduction number $R_{0}$ at the beginning of the epidemic in European countries as being equal to $4.22 \pm 1.69$, with its maximum value 6.33 detected in Germany. By May 10, 2020 this number went down to $0.67 \pm 0.18$. In October there is a new growth in morbidity, that is, the second epidemic wave [10].

Now we estimate SIR model parameters for Israel using data given on Worldometers web-site that allows one to track daily numbers 
of infected, recovered, and dead people [18]. At the beginning of the epidemic starting from March 1 to 30 a number of confirmed infected people $i(t)$ grows from 10 to 5,114 (Figure 3); there are only 244 recovered people over this period, that is, less than $5 \%$. Parameter $\alpha_{1}$, a growth rate for a number of infected persons, is estimated as $0.21 \pm 0.09$; a number of infected ones doubles approximately each three days. This estimate is well in line with figures given for Israel and France [16, 17].

Active Cases

(Number of Infected People)

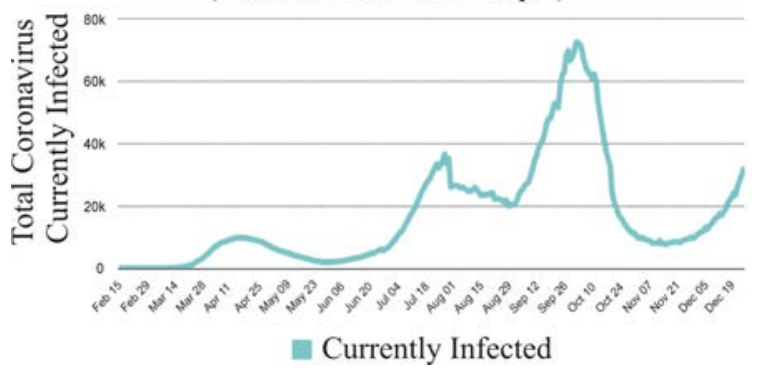

Figure 3. Time dependence for a number of infected $i(t)$ in Israel [8]

Parameter $\alpha_{2}=0.045 \pm 0.03$, recovery rate, is obtained basing on data collected after strict lockdown was introduced from May 4 to 27, when a number of confirmed infected people $i(t)$ amounted to several thousands, and average daily number of new infected people did not exceed 30 . Typical recovery time, that is a time of double decrease in a number of infected amounts 15.5 days. Further we assume that parameter $\alpha_{2}$ does not change during the pandemic.

When quarantine restrictions are eased off, it is accompanied with the $2^{\text {nd }}$ epidemic wave and a number of infected people grows exponentially as per a time depending exponent: June 2-22, 0.04 1/day; June 22-27, 0.045 1/day; June 27 - July 8, 0.079 1/day. Bearing in mind that the exponent characterizing an increase in a number of infected people is $\alpha_{1}-\alpha_{2}$, parameter $\alpha_{1}$ is estimated to be equal to $0.085 ; 0.09$; and 0.124 for these time intervals respectively.

At the beginning of the epidemic $R_{0}$ can be estimated as 4.5 without any quarantine measures or individual or social protection measures being taken. When $R_{0}$ has such a high value, col- lective immunity will be formed only when more than $95 \%$ population have been infected and recovered (Figure 4).

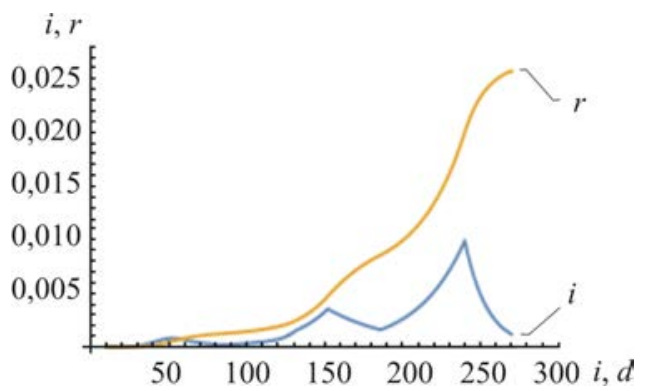

Figure 4. Modeling the pandemic in Israel

This threshold value for collective immunity formation is well in line with predictions made in other works [19]. Some researchers think that $R_{0}$ goes down with time, and this threshold will be substantially lower, however, not lower than several dozen per cent (now registered infected and recovered people account for approximately $3 \%$ of the population) $[10,17,19]$. At the beginning of the $2^{\text {nd }}$ epidemic wave on June $2-22 R_{0}=1.9$; and it is growing as the pandemic develops: June 22-27, $R_{0}=2$; June $27-$ July $8, R_{0}=2.8$.

After July 8 there are strong daily fluctuations that do not allow assessing parameters reliably. In the next part we are planning to use effective values for long time intervals.

1.2. Wave-like pandemic. Results obtained via modeling the pandemic in Israel and the solution to the equations (1)-(3) is given in Figure 3 , and this simulation quite corresponds to morbidity growth (Figure 3). The beginning of the epidemic is characterized with exponential grow thin a number of infected people over time $\sim \operatorname{Exp}(\alpha t)$ prior to strict lockdown was introduced in early April and an exponential decrease in this number in May, $\alpha=\alpha_{1}-\alpha_{2}<0$. Step-bystep quarantine measures cancelling in late May - early June resulted in a new increase in a number of infected people with parameter $\alpha_{1} \approx 0.1$ growing over time. In July and August, when there are vacations in educational establishments, vacations for workers, and still valid quarantine measures, this number goes down. The beginning of the academic year causes a new growth in a number of infected people with $\alpha_{1} \approx 0.09$ and it is only the repeated most strict 
lockdown introduced in late September that leads to the disease spread slowing down.

In Israel in November quarantine measures are being gradually cancelled. We can predict further epidemic development basing on parameter $\alpha_{1}$ values for June-July and September. These values are substantially lower than at the beginning of the pandemic, $0.21 \pm 0.09$. It is due to people wearing face masks in public places, ban on any mass events, distance learning, etc. Figure 5 shows modeling results. As we can see, a slight change in parameter $\alpha_{1}$ results in substantial changes in the results. Thus, at $\alpha_{1}=0.09$ it is predicted that a threshold value for a number of infected people (approximately 75 thousand), when the second lockdown is introduced, is reached in 52 days, and at $\alpha_{1}=0.1$ this threshold value is reached in 42 thousand. Figure 5 shows a range for probable pandemic development.

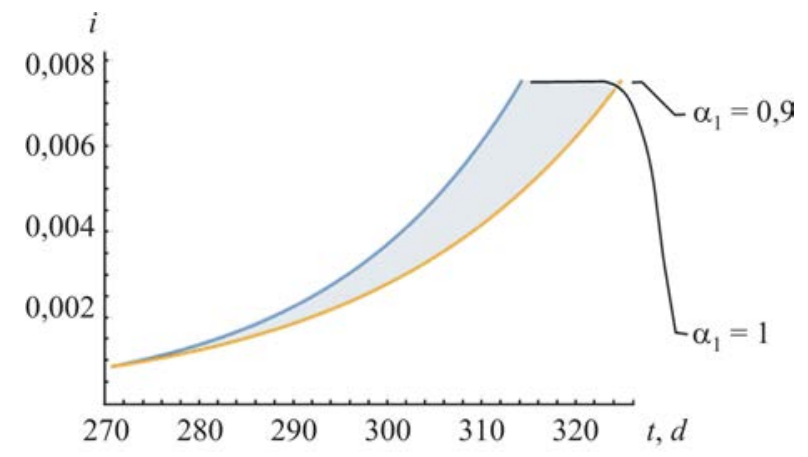

Figure 5. Pandemic prediction

After October 20 some quarantine measures were step-by-step cancelled as nonfood shops and trade centers were opened, children returned to schools, etc. In mid-November there was a new exponential growth (Figure 3) in a number of infected people, and it went up to 17,500 over 20 days; more than 30 thousand, over 30 days; and approximately 49 thousand, over 40 days. Our predictions are well in line with values given in Figure 5.

On December 28 the third lockdown (strict quarantine) is introduced in Israel.

The simplest SIR model contains two parameters $\alpha_{1}$ and $\alpha_{2}$ that depend on many factors (type of a disease, population density, age structure, mobility, etc.) and in more common case on time. These parameters are determined basing on epidemic development in a given country, region, or a city. SIR model allows, assessing significant parameters at least at a first approximation including spread of a disease, burden on medical organizations, necessary number of beds in hospitals, and expected mortality. The latter can be estimated as relevant shares of infected people. However, this model does not take into account an actual clinical course of a disease and such factors as incubation, the illness itself, various clinical courses of a disease, age-related differences, etc. Several more realistic models were developed that allow one to account the abovementioned and other factors as well as specific peculiarities of various diseases [4-12, 17, 20, 21]. These models are based on an assumption that susceptible, infected, and recovered people are distributed evenly over space and a pandemic is described with finding solutions to usual differential equations of (1)-(3) type. However, in reality infected people, even in such a small country as Israel, are distributed extremely unevenly and their distribution is not proportionate to population density. Even on large cities there are districts with different morbidity levels. There are several models that take into account uneven distribution of susceptible, infected, and other people [2, 21-23]. Reaction-diffusion epidemiologic models belong to this category $[2,4,7,22]$.

2. Reaction epidemiologic model. Mathematical description of reaction-diffusion epidemiologic models contains a system of equations in partial derivatives similar to equations that describe diffusion with a chemical reaction $[2,4,7,22]$. A number of equations and a type of members that correspond to are action depend on a chosen model for infection transfer, number of examined population groups distinguished as per age and activity (schoolchildren, working population, retired people etc.). Reaction-diffusion model parameters such as diffusion coefficients characterize mobility of a given population group. Some authors take into account natural changes in population number due to birthrate and mortality as well as mobility of recovered people who have immunity [22]. However, this population group does not influence dynamics of a disease and can be neglected when an epidemic is analyzed. 
Most research works dwell on onedimensional models but we are going to concentrate on a more realistic two-dimensional one $[2,22]$. Let us start with a simple twodimensional model based on SIR model that allows obtaining several analytic solutions along with numerical ones:

$$
\begin{gathered}
\frac{\partial s_{n}}{\partial t}=a_{s}\left(\frac{\partial^{2} s_{n}}{\partial x^{2}}+\frac{\partial^{2} s_{n}}{\partial y^{2}}\right)-\alpha_{d 1} s_{n} i_{n} \\
\frac{\partial i_{n}}{\partial t}=a_{i}\left(\frac{\partial^{2} i_{n}}{\partial x^{2}}+\frac{\partial^{2} i_{n}}{\partial y^{2}}\right)+\alpha_{d 1} s_{n} i_{n}-\alpha_{d 2} i_{n}
\end{gathered}
$$

where $a_{s}$ and $a_{i}$ are the parameters that characterize mobility of susceptible and infected people, respectively, $s_{n}$ and $i_{n}$, are their normalized densities in an examined area. Let us note that density of recovered people $\left(r_{n}\right)$ can be determined via finding solutions to equations (8), (9) and

$$
\frac{\partial r_{n}}{\partial t}=a_{i}\left(\frac{\partial^{2} r_{n}}{\partial x^{2}}+\frac{\partial^{2} r_{n}}{\partial y^{2}}\right)+\alpha_{d 2} r_{n}
$$

Let us assume that parameters $a_{s}, a_{i}, \alpha_{d 1}$, and $\alpha_{d 2}$ do not depend on time and coordinates.

It is convenient to use dimensionless variables: $\tau=a_{s} t, \xi=x \sqrt{\frac{\alpha_{d 1}}{a_{s}}}, \eta=y \sqrt{\frac{\alpha_{d 1}}{a_{s}}}$ in equations (8)-(9); the equations are rewritten as:

$$
\begin{gathered}
\frac{\partial s_{n}}{\partial \tau}=\frac{\partial^{2} s_{n}}{\partial \xi^{2}}+\frac{\partial^{2} s_{n}}{\partial \eta^{2}}-s_{n} i_{n}, \\
\frac{\partial i_{n}}{\partial \tau}=\frac{a_{i}}{a_{s}}\left(\frac{\partial^{2} i_{n}}{\partial \xi^{2}}+\frac{\partial^{2} i_{n}}{\partial \eta^{2}}\right)+s_{n} i_{n}-\frac{\alpha_{d 2}}{\alpha_{d 1}} i_{n}
\end{gathered}
$$

In these equations there are two parameters that characterize spread of a disease: the first one $\frac{a_{i}}{a_{s}}$ is a ratio of values that characterize mobility of infected and susceptible people (should not exceed 1); the second one, $\frac{\alpha_{d 2}}{\alpha_{d 1}}$, is an inverse value of $R_{0}$ analogue. Boundary conditions depend on what surrounds the considered territory. When it comes down to Israel and some other countries with relatively small squares that imposed strict limitations on mov- ing into and out of country people, as well as to some remote Russian regions, we can believe that a flow of infected and susceptible people across a border $\Gamma$ is equal to zero, that is:

$$
\left.\frac{\partial s_{n}}{\partial \vec{n}}\right|_{\Gamma}=0 \text { and }\left.\frac{\partial i_{n}}{\partial \vec{n}}\right|_{\Gamma}=0,
$$

where $\vec{n}$ is a single vector which is normal to boundary $\Gamma$.

Figure 5 shows how an epidemic develops in a square area $\{-4<\xi<4 ;-4<\eta<4\}$ with initial conditions $\quad i_{n}(0, \xi, \eta)=0.0001 \operatorname{Exp}\left[-20\left(\xi^{2}+\eta^{2}\right)\right]$ and $S_{n}=1$ in the whole area. All calculations used in the present work were accomplished with Mathematica 12.

As we can see, when $\tau \geq 5$, infected people are distributed practically evenly, and a model belonging to SIR type similar to that described in the previous section can be applied in further analysis of an epidemic development. For the given initial and boundary conditions, a time needed for reaching a uniform distribution of infected people is estimated as several (3-5) characteristic diffusion time $\tau_{x}=\frac{L^{2}}{\pi^{2}}$, where $L$ is a half of a square side.

People who were infected with coronavirus infection account for a relatively small share of the total population, therefore COVID-19 epidemic can be assumed to be at its initial stage. Non-uniform spatial distribution of infected people at this stage-in the epidemic that is observed in many countries and even in large cities is due to uniform distribution of infected people not being reached yet, and non-uniform distribution of susceptible people, and also parameters $a_{s}, a_{i}, \alpha_{d 1}$, и $\alpha_{d 2}$ being dependent on time and coordinates (see the next section).

The considered example (Figure 6) has a certain peculiarity that is a decrease in a number of infected people in the center, despite $\frac{\alpha_{d 1}}{\alpha_{d 2}}>1$. To explain this phenomenon, let us consider an approximation that allows obtaining an analytical solution.

At an initial stage in an epidemic, just as it is the case with COVID-19, we can assume that density of susceptible people changes only 

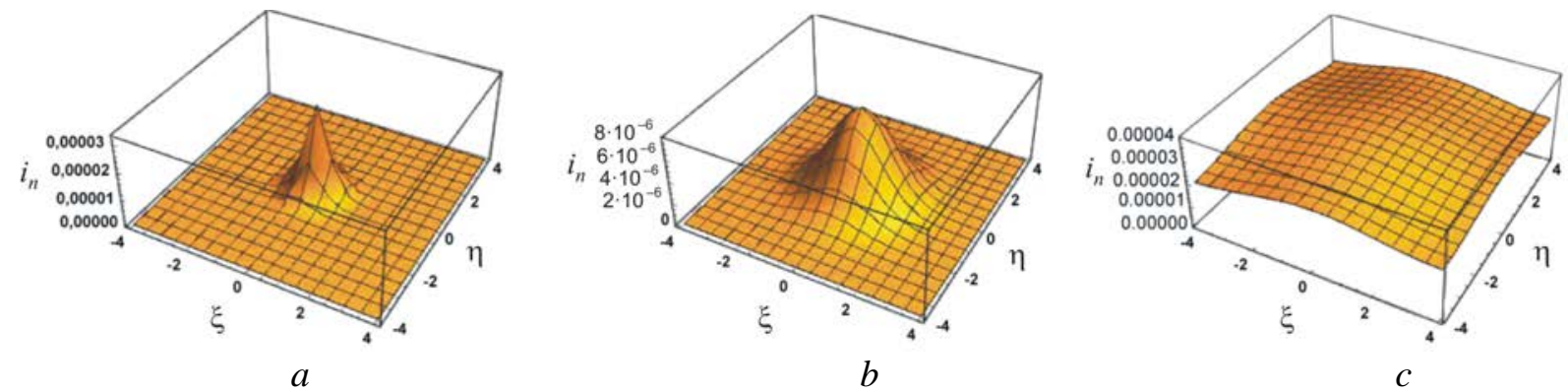

Figure 6. Epidemic development in a square area $16 \times 16$ at $\frac{a_{i}}{a_{s}}=1, \alpha_{d 1}=0.22$, and $\alpha_{d 2}=0.048$ : spatial distribution of infected people: a) at $\tau=0.1$; b) at $\tau=0.5$ and c) at $\tau=5$

slightly and does not depend on coordinates, i.e. $S_{n}=$ const $=1$. In this case, spread of a disease is described with a solution to the following equation:

$$
\frac{\partial i_{n}}{\partial t}=a_{i}\left(\frac{\partial^{2} i_{n}}{\partial x^{2}}+\frac{\partial^{2} i_{n}}{\partial y^{2}}\right)+\left(\alpha_{d 1}-\alpha_{d 2}\right) i_{n}
$$

If

$$
\text { we }
$$

replace $i_{n}=\tilde{i}_{n}(t, x, y) \exp \left[\left(\alpha_{d 1}-\alpha_{d 2}\right) t\right]$ to solve the equation (12), we get a new equation for $\tilde{i}_{n}(t, x, y)$

$$
\frac{\partial \tilde{i}_{n}}{\partial t}=a_{i}\left(\frac{\partial^{2} \tilde{i}_{n}}{\partial x^{2}}+\frac{\partial^{2} \tilde{i}_{n}}{\partial y^{2}}\right)
$$

Analytical solutions for the equation (13), depending on boundary conditions type, a shape of a boundary, and initial conditions, are given, for example, in a Handbook on linear equations in mathematical physics ${ }^{1}$. At an initial stage in an epidemic infected people are close to the first infected ones, and in this case we can use an approximation for an infinite range $\{-\infty<x<\infty ;-\infty<y<\infty\}$ and assume that at an initial moment of time $t=0$ infected people are uniformly distributed in an area given as $\left\{-x_{0}<x<x_{0} ;-y_{0}<y<y_{0}\right\}$. The initial distribution is

$i_{n}(0, x, y)=\left\{\begin{array}{cc}i_{n 0} & \text { at }|x|<x_{0} \text { and }|y|<y_{0}, \\ 0 & \text { at }|x| \geq x_{0} \text { and }|y| \geq y_{0} .\end{array}\right.$

The solution to equation (12)

$$
\begin{gathered}
i_{n}(t, x, y)= \\
=\frac{i_{n 0}}{4}\left[\operatorname{erf}\left(\frac{x_{0}-x}{2 \sqrt{a_{i} t}}\right)+\operatorname{erf}\left(\frac{x_{0}+x}{2 \sqrt{a_{i} t}}\right)\right] \\
{\left[\operatorname{erf}\left(\frac{y_{0}-y}{2 \sqrt{a_{i} t}}\right)+\operatorname{erf}\left(\frac{y_{0}+y}{2 \sqrt{a_{i} t}}\right)\right]} \\
\exp \left[\left(\alpha_{d 1}-\alpha_{d 2}\right) t\right]
\end{gathered}
$$

where $\operatorname{erf}(x)$ is the probability integral. Let us note that analytical solutions are more complicated for other initial and boundary conditions, however, their qualitative behavior is similar.

Integrating (14) over the whole area, we obtain time dependence for the total number of infected people

$$
i(t)=4 i_{n 0} x_{0} y_{0} e^{\left(\alpha_{d 1}-\alpha_{d 2}\right) t}
$$

Accounting that $4 i_{n 0} x_{0} y_{0}=i_{0}$ is the normalized initial number of infected people and comparing solutions to (6) and (15), we can conclude that $\alpha_{d 1}=\alpha_{1}$ and $\alpha_{d 2}=\alpha_{2}$; these parameters and their dependence on time can be estimated with the SIR model. As well within the SIR model, an epidemic ends up at $\alpha_{1}<\alpha_{2}$, although a disease spreads across the considered area and occurs in new districts.

When performing qualitative analysis, we take $y_{0}=x_{0}$ and in the center of the area $\{x=0 ; y=0\}$ density of the infected people is

$$
i_{n}(t, 0,0)=i_{n 0} e r f\left(\frac{x_{0}}{2 \sqrt{a_{i} t}}\right)^{2} \exp \left[\left(\alpha_{1}-\alpha_{2}\right) t\right]
$$

\footnotetext{
${ }^{1}$ Polyanin A.D. Handbook on linear equations in mathematical physics. Moscow, Phizmatlit Publ., 2001, 576 p. (in Russian).
} 
In expression (16) erf decreases with time, and exp grows. It is convenient to replace $t$ with $\tilde{t}=\frac{4 a_{i}}{x_{0}^{2}} t$, then dependence of infected people density in the area center is characterized with just one parameter $\gamma=\frac{x_{0}^{2}}{4 a_{i}}\left(\alpha_{1}-\alpha_{2}\right)$ :

$$
i_{n}(t, 0,0)=i_{n 0} \operatorname{erf}\left(\frac{1}{\sqrt{\tilde{t}}}\right)^{2} \exp [\gamma \tilde{t}] .
$$

Depending on $\gamma$ value, two scenarios are possible (Figure 7): the first is a monotonous growth with time $(\gamma=0.7)$ and the second one is a slight growth in $i_{n}(t, 0,0)$, and then a decrease with time $(\gamma=0.1)$. These scenarios are divided by $\gamma \approx 0.52$. The-first scenario can become a reality when an epidemic starts in a densely populated area (the beginning of COVID-19 epidemic in China); the second, when a disease is brought into a country and starts from an airport, railway station, etc. At any $\gamma$ values and longtime intervals $i_{n}(t, 0,0)$ grows proportionate to $\frac{1}{t} \exp \left[\left(\alpha_{1}-\alpha_{2}\right) t\right]$. This analysis provides a qualitative description of the numeric results given in Figure 7.

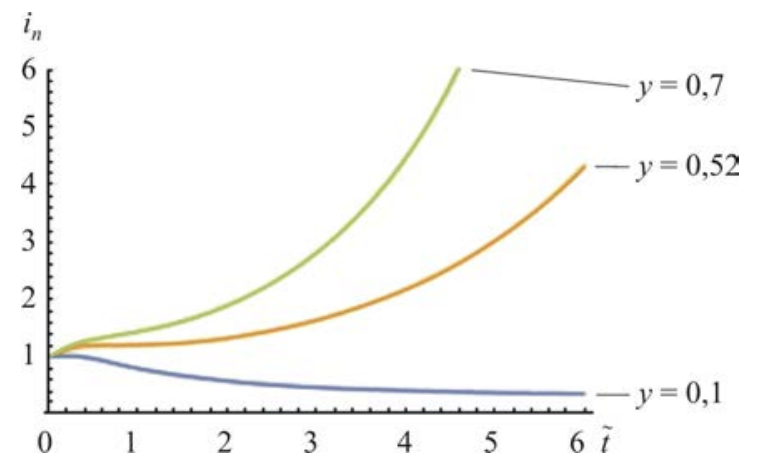

Figure 7. Initial stage of an epidemic: time dependence of a number of infected people in the center of contagion

It is convenient to analyze how a disease spreads in an assumed axially symmetrical spread. On one hand, spread of a disease is close to axially symmetrical one in certain regions and cities. At an initial stage it is so even is a square area (Figure 8). On the other hand, such an approximation being considered allows illustrating the results more clearly. The equations (8) and (9) are rewritten as

$$
\begin{gathered}
\frac{\partial s_{n}}{\partial t}=a_{s} \frac{\partial}{\partial \rho}\left(\rho \frac{\partial s_{n}}{\partial \rho}\right)-\alpha_{1} s_{n} i_{n} \\
\frac{\partial i_{n}}{\partial t}=a_{i} \frac{\partial}{\partial \rho}\left(\rho \frac{\partial i_{n}}{\partial \rho}\right)+\alpha_{1} s_{n} i_{n}-\alpha_{2} i_{n}
\end{gathered}
$$

with boundary

$$
\begin{aligned}
& \left.\frac{\partial i_{n}}{\partial \rho}\right|_{\rho=0}=0 ;\left.\frac{\partial i_{n}}{\partial \rho}\right|_{\rho=\rho_{m}}=0 ; \\
& \left.\frac{\partial s_{n}}{\partial \rho}\right|_{\rho=0}=0 ;\left.\frac{\partial s_{n}}{\partial \rho}\right|_{\rho=\rho_{m}}=0 ;
\end{aligned}
$$

and initial

$$
\begin{gathered}
S_{n}(0, \rho)=1 ; \\
i_{n}(0, \rho)=0.04 \operatorname{Exp}\left[-\rho^{2} / 0.01\right]
\end{gathered}
$$

conditions.

Here $\rho=\sqrt{x^{2}+y^{2}}, \quad \rho_{m}$ is the external boundary radius for the considered area; it is assumed that there are no people moving into and out of it; numeric parameters 0.04 and 0.01 in initial conditions for $i_{n}(20)$ correspond to an initial number of infected people equal to $10^{-6}$ from the total population in the area. Results of simulation of spatio-temporal epidemic development are presented in Figure 8.

For each radius the time dependences of numbers of susceptible and infected people are similar to these presented in Figure 1. However, the maximum number of infected and time necessary to reach it, just like in SIR model, depend on $\alpha_{1}, \alpha_{2}$ and their ratio as well as on a radius, values of parameters $a_{1}$ and $a_{2}$ (please, compare Figures $8 \mathrm{~b}$ and $8 \mathrm{c}$ ), as well as their ratio (please, compare Figures $8 \mathrm{a}$ and $8 \mathrm{~b}$ ). The same time dependence exists for integral characteristics, the total numbers of susceptible, infected and recovered people in the whole area (Figure 9).

Despite susceptible people are distributed uniformly at an initial time, the maximum density of infected people decreases as a distance from the diseases focus grows (Figure 8, 10a). However, a number of infected people per a unit of a radius length $i_{n r}(t, \rho)=2 \pi \rho i_{n}(t, \rho)$ is 


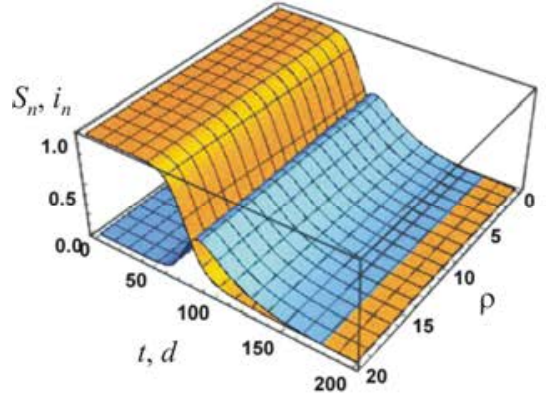

$a$

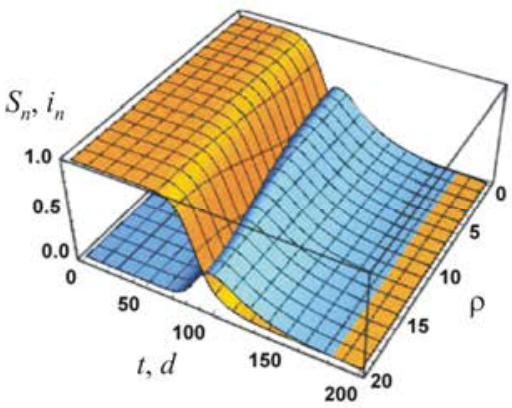

$b$

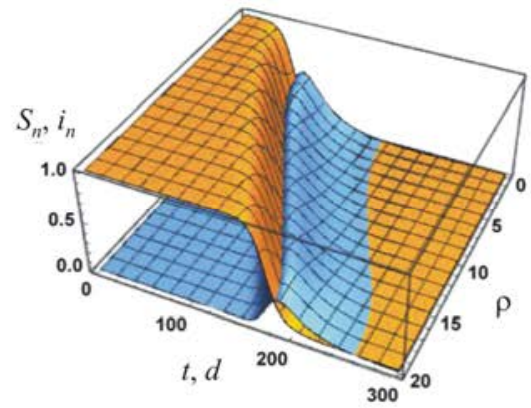

$C$

Figure 8. Spatio-temporal epidemic development; yellow color shows normalized density of susceptible people $s_{n}$, blue color - infected $i_{n}$ : a) $a_{s}=1$ and $a_{i}=1$; b) $a_{s}=1$ and $a_{i}=0.3$; c) $a_{s}=0.1$ and $a_{i}=0.03$. In all cases $\alpha_{1}=0.22, \alpha_{2}=0.048$ and $\rho_{m}=20 ; a_{s}$ and $a_{i}$ are values normalized by the actual (dimension) coefficient of the susceptible people mobility $\lambda$ for the case a), and distance $\rho$ is given in units $\sqrt{\lambda}$

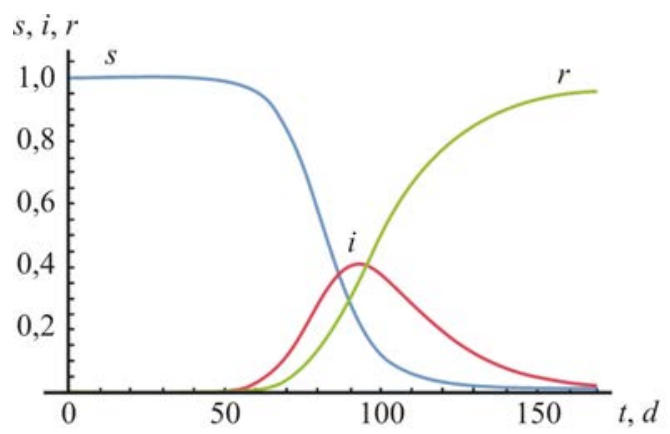

Figure 9. Dependence for a number of susceptible, infected, and recovered people in the whole area.

Parameters are taken just as for Figure 8b, distribution of recovered people was determined by solution to equation of a type (17), in which the last member to the right in it being replaced by $+\alpha_{2} i_{n}$, with zero initial conditions

increasing with a distance from the center (Figure 10b). Therefore, at the same social conditions (population density, mobility of susceptible and infected people, the same parameters that characterize infection and recovery rates) and in spite of active migration to the center, number of infected people will be substantially higher at the periphery than at the center.

The higher density of infected people at the center is caused by the following: at the beginning of an epidemic density of susceptible $(\rho \leq 1)$ close to a disease focus goes down due to their moving into «infected» group. At the same time this density practically stays the same in remote areas where infection has not yet occurred. Within the model this situation
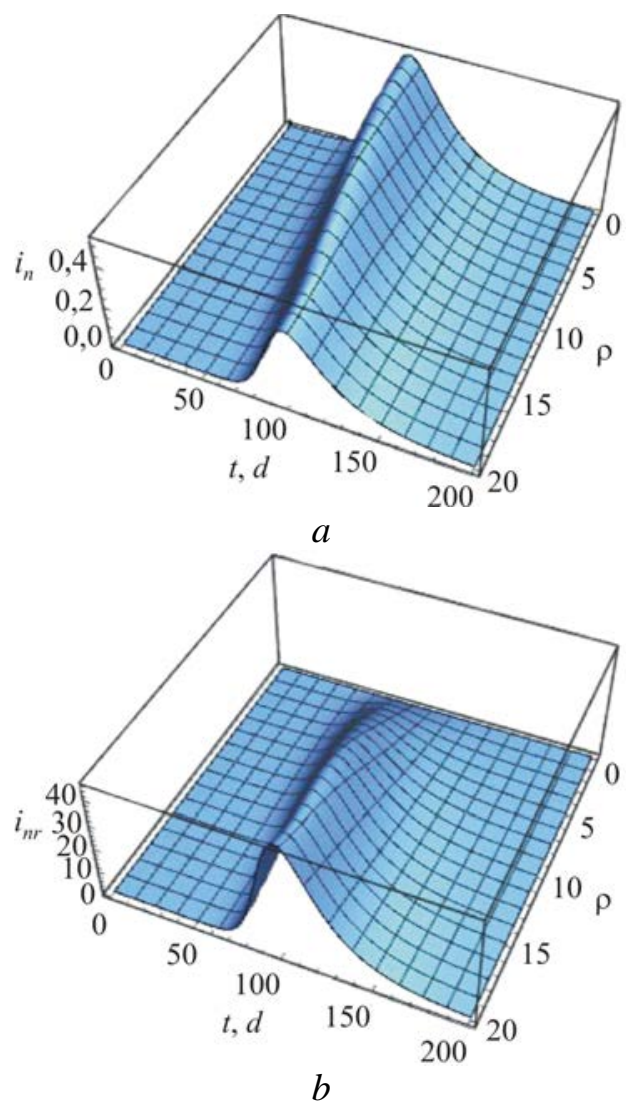

Figure 10. Density of infected people a) and a number of infected ones per a unit of a radius length $b$ ) for the case shown in Figure 8b

results in susceptible people moving to the center. For the case shown in Figure $7 b$, in an area limited by $\rho=1$, and for 200 days ( $t=200$ days) $\sim 1,1 N /\left(\pi \rho_{m}^{2}\right) \quad-$ susceptible people migrate more than $1 / 3$ initially being 
there $\pi N /\left(\pi \rho_{m}^{2}\right)$; for the case shown in Figure $7 \mathrm{~b}$, a number of migrated people is several times higher than $\pi N /\left(\pi \rho_{m}^{2}\right)$. A model based on equations (8) and (9) can be applied only with certain limitations for describing spread of a disease in case susceptible people are distributed non-uniformly. Even in case there is no disease, the solution to equation (8) describes reaching uniform distribution of susceptible people regardless of their initial distribution.

3. Heterogeneous reaction-diffusion epidemiologic model. Population migration can be divided into two types: long-distance travels to other cities, regions, or countries and every day trips (home-work, studies, shopping, etc.). The first type involves a disease being carried over long distances and it is random in its nature. Such spread of a disease can be prevented by imposing limitations on such travels (limited and/or cancelled flights, closing a country completely, etc.), as well as via introducing sanitary-epidemic measures: preliminary diagnostics and quarantine after arrival in a country. These measures were taken in Israel and some other countries at the beginning of COVID-19 epidemic. In countries with large territories (Russia, China, Canada, and the USA) such measures are to be taken in specific regions and areas.

Daily migration occurs within a settlement, in case of a megacity including its satellites (for example, Moscow, Tel-Aviv, or New-York). In the morning people flows go into downtown with high population density; the situation is inverse in the evening. The characteristic epidemiologic times $1 / \alpha_{1}$ and $1 / \alpha_{2}$ are significantly longer than a characteristic time of daily migration that is equal to approximately half a day. It allows introducing averaged density of susceptible people as

$$
\tilde{s}_{n}(t, r)=s_{n}(t, r)+\Delta s_{n}(t, r) / 2
$$

where $s_{n}(t, r)$ is the density of people who permanently live in a given district, and $\Delta s_{n}(t, r)$ is the density of migrating people. The latter value can be both positive for down- town and negative for periphery. At an initial time of an epidemic

$$
\int_{\Omega}\left[s_{n}(0, r)+\Delta s_{n}(0, r)\right] d \Omega=N
$$

where $\Omega$ is the square of the considered area. Further we assume that density of susceptible people changes only due to their moving into the infected group. Equations (8) and (9) are given as follows

$$
\frac{\partial \tilde{s}_{n}}{\partial t}=-\alpha_{1} \tilde{s}_{n} i_{n}
$$

$\frac{\partial i_{n}}{\partial t}=\frac{\partial}{\partial x}\left(a_{i x} \frac{\partial i_{n}}{\partial x}\right)+\frac{\partial}{\partial y}\left(a_{i y} \frac{\partial i_{n}}{\partial y}\right)+\alpha_{1} \tilde{s}_{n} i_{n}-\alpha_{2} i_{n}$

and coefficients $\alpha_{1}, \alpha_{2}, a_{i x}$, and $a_{i y}$ depend on time and coordinates, and coefficients $a_{i x}$ and $a_{i y}$ that characterize mobility of infected people generally depend also on a direction. Let us note that if susceptible people are assumed to be immobile, coefficients $a_{i x}$ and $a_{i y}$ rather characterize local spread of a disease and not mobility of an infected people.

3.1. Influence exerted by lockdown on spatio-temporal spread of a disease. Both the local and integral maximum numbers of infected people account of dozens per cent of population (Figures 1, 8, and 9). In order to decrease a number of infected and dead people as well as to prevent medical aid collapse, limitations and lockdowns are introduced, when a share of infected people is still insignificant, and we can assume that $\tilde{s}_{n}$ depends only on implemented limitations and any changes in it due to contagion are negligible. In this case changes in $\tilde{s}_{n}$ are due to changes in $\Delta s_{n}(t, \rho)$, caused by limitations or lockdown. We will assume that coefficients $\alpha_{1}, a_{i x}=a_{i y}$ depend only on time, $s_{n}(t, \rho)=1$ and parameter $\alpha_{2}$, that characterizes the recovery rate is constant. For the axially symmetrical approximation within the introduced assumptions, epidemic development is described with a solution to the equation

$$
\begin{aligned}
& \frac{\partial i_{n}}{\partial t}=a_{i}(t) \frac{\partial}{\partial \rho}\left(\rho \frac{\partial i_{n}}{\partial \rho}\right)+ \\
& +\alpha_{1}(t, \rho) \tilde{s}_{n}(t, \rho) i_{n}-\alpha_{2} i_{n}
\end{aligned}
$$


with boundary (19) and initial (20) conditions.

Lockdown introduction leads to lower population mobility, regarding both susceptible and infected people, as well as their meetings and, consequently, contagion among susceptible being less probable. To demonstrate lockdown efficiency, we assume that:

- lockdown is introduced at $t=25$ days, the total number of infected people is approximately $0.06 \%$ (the first lockdown was introduced in Israel when a number of infected people was approximately $0.1 \%$ ) and is canceled at $t=55$ days;

- after lockdown is canceled, all model parameters return to their initial values;

- efficient distribution of susceptible people density $\tilde{s}_{n}$ is given as

$$
\tilde{s}_{n}=\frac{b(t) \exp \left[b(t) \rho_{m}^{2}\right]}{\exp \left[b(t) \rho_{m}^{2}\right]-1} \rho_{m}^{2} \exp \left[-b(t) \rho^{2}\right]
$$

- lockdown is characterized with a decrease in parameters $a_{i}$ from 0.3 to 0.1 ; $\alpha_{1}$ from 0.22 to 0.02 and $b \rho_{m}^{2}$ from 0.693 to 0.1054 (dependence of susceptible density on a radius prior to and during a lockdown is shown in Figure 11);

- as it was above, $\alpha_{2}=0.048$ and $\rho_{m}=20$.

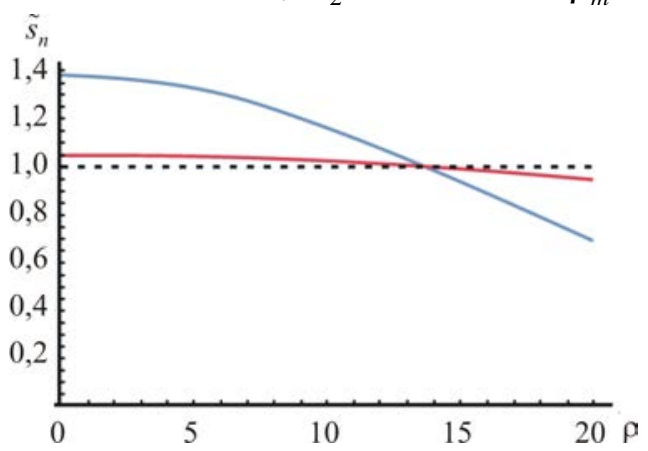

Figure 11. Dependence of susceptible people density on a radius prior to (blue curve) and during lockdown (red curve); green dotted line corresponds to even distribution

Computation results are presented in Figure 12.

In the considered range density of infected people does not exceed $2 \%$ of the population density; it justifies our assumption that changes in density of susceptible people can be neglected. For each radius time dependence for density of infected people is qualitatively similar to the same dependence for the total number of infected ones (Figure 5). There is a growth in a number of infected people that is close to exponential prior to lockdown introduction, a decrease in a number of infected people during lockdown and a drastic rise after lockdown has been canceled and population got back to normal life (a wave-like epidemic). However, at $\rho>15$ density of infected people, both prior to and during lockdown, is finite but also negligible, approximately $10^{-6}$, it can be lower than a single infected person per one unit area. It indicates that lockdown introduction in the area $\rho>15$ is an excessive measure that does unjustified damage. Spread of a disease in this area is a drawback typical for models based on solving equations similar to heat conductivity ones: a paradox of an infinite rate at which heat spreads; when it comes down to an epidemic, it is a paradox related to an infinite disease spread rate.

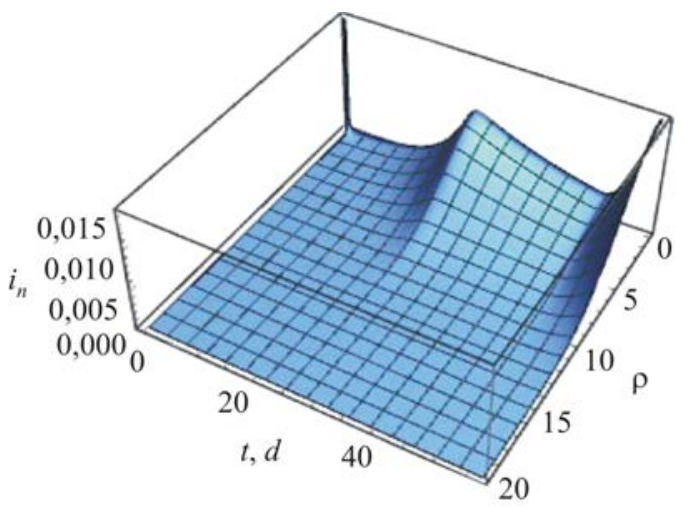

Figure 12. Spatio-temporal distribution of infected

4. Reaction-diffusion epidemiologic model with finite contagion time. In the previous sections we applied parabolic equations to describe spatio-temporal spread of a disease; these equations have a physically paradoxical property, namely infinite rate at which disturbance spreads $[24,25]$. When it comes down to epidemiologic models, it means that a disease spreads instantly: at an area boundary at $\rho=20$ a reaction-diffusion model instantly gives insignificant but still finite density of infected people. It results from a non-apparent assumption that infection spreads from infected to susceptible persons immediately thus making the latter move into the «Infected» category. In actual conditions a disease process is more complicated and can consist of at least two stages: incubation (an infected person is not a source of contagion 
during a part of this period and has no apparent clinical signs of a disease) and a disease itself. SEIR model (Susceptible-Exposed-InfectedRemoved model) allows taking incubation into account $[9,10,17,20]$. Three states considered within the SIR model are added with one more state, Exposed or infected in their incubation period. To introduce this state into the system of equations (8)-(9) we should add one more nonlinear parabolic equation. However, this approach does not solve an issue related to infinite disease spread rate. To resolve the issue, we suggest using Cattaneo-Vernotte model [24, 25] that leads to a finite spread rate. Within the model relaxation (delay) time $\tau_{\text {rel }}$ is introduced and in general case the system of equations (8)-(9) can be represented as

$$
\begin{gathered}
\frac{\partial s_{n}}{\partial t}=\frac{\partial}{\partial x}\left(a_{s x} \frac{\partial s_{n}}{\partial x}\right)+ \\
+\frac{\partial}{\partial y}\left(a_{s y} \frac{\partial s_{n}}{\partial y}\right)-\alpha_{d 1} s_{n} i_{n} \\
\tau_{r e l} \frac{\partial^{2} i_{n}}{\partial t^{2}}+\frac{\partial i_{n}}{\partial t}=\frac{\partial}{\partial x}\left(a_{i x} \frac{\partial i_{n}}{\partial x}\right)+ \\
+\frac{\partial}{\partial y}\left(a_{i y} \frac{\partial i_{n}}{\partial y}\right)+\alpha_{d 1} s_{n} i_{n}-\alpha_{d 2} i_{n}
\end{gathered}
$$

with its parameters depending on time and coordinates.

Equation (27) is a hyperbolic one that's allows obtaining a finite rate of disturbance spread and is widely used for solving heat and diffusion tasks [24, 25]. This equation contains the second time derivative; to find numerical solution it is necessary to set an additional initial condition to all mentioned above. Relaxation time $\tau_{\text {rel }}$ is a characteristic showing the process disequilibrium and it takes into account flow inertia; in our case it is a delay (inertia) in contagion. At an initial moment of time we can consider that

$$
\left.\frac{\partial i_{n}(t, x, y)}{\partial t}\right|_{t=0}=0
$$

Solution to the system of the equations (26) and (27) in axially symmetry is given in Figure 13.

The solution is similar to computations obtained for the model based on parabolic equations (17) and (18) (Figure 8). However, when a delay in contagion is taken into account, it leads to slower disease spread and a front of a disease spread occurs, that is, a moving frontier between an area where there are infected people and an area where such people are absent. Density of infected people goes down to zero smoothly as this frontier is getting closer and $i_{n}=0$ beyond it. This is the principal difference between two solutions; it is shown in Figure 14.

A decrease in $\tau_{\text {rel }}$ approximates solution to the equations (26) and (27) to computations made for equations (17) and (18). However, there is a frontier even for small $\tau_{\text {rel }}$ values: at $\rho_{b} \approx 12$ for $\tau_{r e l}=1$ and $\rho_{b} \approx 15$ for $\tau_{\text {rel }}=0.1$. Predicting how this frontier is going to move allows scientific substantiation for gradual lockdown introduction.

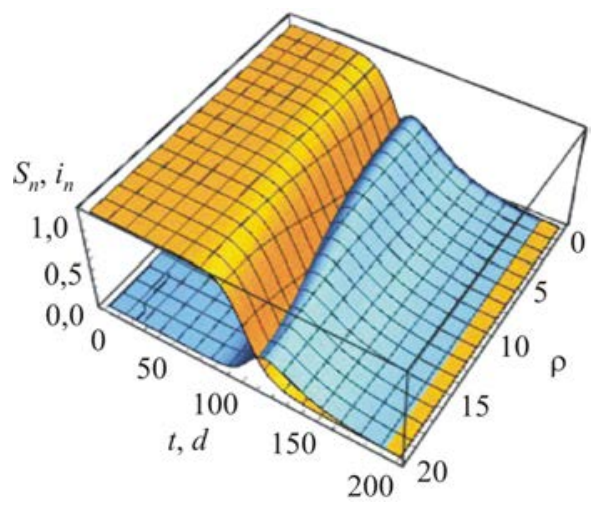

Figure 13. Simulating an epidemic taking into account finite contagion period. Initial and boundary conditions are taken from (19), (20), and (28); the parameters were taken as constant: $a_{s}=1, a_{i}=0.3, \alpha_{1}=0.22, \alpha_{2}=0.048$, and $\tau_{\text {rel }}=1$

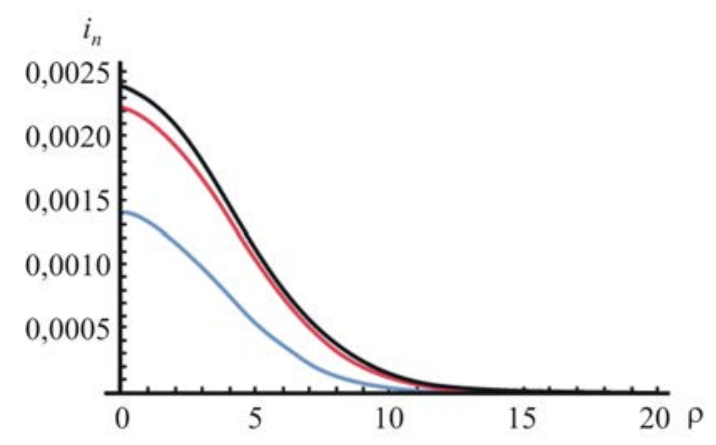

Figure 14. Spatial distribution of infected people at $t=30$ : blue curve means $\tau_{\text {rel }}=1$;

red curve, $\tau_{r e l}=0.1$; black curve, $\tau_{r e l}=0$ (solution that does not take delay into account) 
Conclusion. In this work we have analyzed spatio-temporal epidemic development and influence exerted by lockdown introduction on spread of a disease. Discussed reaction-diffusion epidemiologic models allow taking into account non-uniform population distribution, changes in population mobility and frequency of contacts between susceptible and infected people due to quarantine measures. It was shown that parameters that characterized infection and recovery rates could be estimated with simpler models belonging to SIR type. A reaction-diffusion model that has been modified in this work allows taking into account daily migration of population and its dependence on time.

We suggested a spatio-temporal model in this work that took into account a delay in contagion (incubation period when an infected person is not a contagion source); the model revealed a possibility that the front of a disease spread might occur.

Model parameters depend on a country; in countries with large territories, on their part; they can be different in different districts in a megacity. These parameters depend on many factors such as population density, population mobility, age groups, an extent to which population is involved into occupational activities, and types of these activities. Correct assessment of these parameters depends on statistic data being complete and reliable, that is correct diagnostics, proper accounting of infected people, etc. Thus, statistics ranks only those among infected people who applied for medical aid to medical organizations and had the diagnosis confirmed by medical experts. Statistical data do not cover infection carriers, patients without any symptoms and patients with a disease in its mild form who didn't apply for medical aid. A study that focused on antibodies occurrence revealed that a number of such infected people who spread a disease could be several times higher than it is stated by official statistics [16, 26].
Efficiency of personal protective equipment (face masks and gloves) and quarantine measures to a great extent depends on how disciplined people are; and variations in model parameters that occur due to limitation being introduced can change considerably over time and depend on a location.

Correct prediction of an epidemic development becomes more vital at the moment due to new and more contagious COVID-19 virus strains occurring in England, the SAR, and some other countries.

Just as this work was being accomplished and prepared for publication, a number of infected people grew and new limitations were introduced in some countries. However, an assumption used in this paper that a number of susceptible people and their density have changed only slightly is still valid.

In December 2020 vaccination started in several countries. As the equation (2) shows, vaccination will be successful when $R_{0} s(t)<1$ is reached, that is, a share of insusceptible people (recovered and vaccinated) is higher than $\left(1-1 / R_{0}\right) \cdot 100 \%$. At $R_{0}=4.5$ the threshold amounts to $78 \%$. In case insusceptible people account for such a share of population, any occurred disease focus will end up without taking any quarantine measures. Quarantine measure introduction and adherence to them results in a decrease in $R_{0}$ and a minimum number of vaccinated necessary for an epidemic to stop. However, cancellation of such measures and a local occurred disease focus will lead to a new epidemic wave.

Funding. This research was supported by the joint grant from the Ministry of Science \& Technology (MOST, № 3-16500), Israel \& Russian Foundation (RFBR, № 19-515-06001).

Conflict of interest. The authors declare there is no any conflict of interests.

\section{References}

1. Mallapaty S. Why COVID outbreaks look set to worsen this winter. Nature, 2020, no. 586, pp. 653 . DOI: 10.1038/d41586-020-02972-4

2. Berrai I.E., Bouyaghroumni J., Namir A. Numerical simulation of spatio-temporal model: case of SIR epidemic model. International Journal of Computer Science Issue, 2014, vol. 11, no. 2, pp. 105-108.

3. Capasso V. Mathematical Structures of Epidemic Systems. Berlin, Springer Publ., 1993, 283 p.

4. Brauer F., Van den Driessche P., Wu J. Mathematical Epidemiology. Berlin, Springer Publ., 2008, 408 p.

5. Bashabsheh M.M., Maslinkov B.I. Simulation modeling of the spatial spread of epidemics (cholera for example) using the method of cellular automatalusing the Anylogic. Internet-zhurnal «Naukovedenie», 2013, no. 6, pp. 1-13 (in Russian).

6. Brauer F., Castillo-Chavez C., Feng Z. Mathematical Models in Epidemiology. Berlin, Springer Science+Business Media Publ., 2019, 619 p. 
7. Al-Showaikh F.N.M., Twizell E.H. One-dimensional measles dynamics. Applied Mathematics and Computation, 2004, vol. 152, no. 1, pp. 169-194. DOI: 10.1016/S0096-3003(03)00554-X

8. Al-Raeei M. The basic reproduction number of new coronavirus pandemic with mortality for India, the Syrian Arab Republic, the United States, Yemen, China, France, Nigeria and Russia with different rate of cases. Clinical Epidemiology and Global Health, 2020, vol. 9, pp. 147-149. DOI: 10.1016/j.cegh.2020.08.005

9. Matveev A.V. The mathematical modeling of the effective measures against the COVID-19 spread. Natsional'naya bezopasnost' i strategicheskoe planirovanie, 2020, vol. 29, no. 1, pp. 23-39 (in Russian).

10. Linka K., Peirlinck M., Kuhl E. The reproduction number of COVID-19 and its correlation with public health interventions. Computation Mathematics, 2020, vol. 7, pp. 1035-1050. DOI: 10.1101/2020.05.01.20088047

11. Ejigu B.A., Asfaw M.D., Cavalerie L., Abebaw T., Nanyingi M., Baylis M. Assessing the impact of non-pharmaceutical interventions (NPI) on the dynamics of COVID-19: A mathematical modelling study in the case of Ethiopia. medRxiv, 2020, 30 p. DOI: 10.1101/2020.11.16.20231746

12. Gross B., Zheng Z., Liu S., Chen X., Sela A., Li J., Li D., Havlin S. Spatio-temporal propagation of COVID-19 pandemics. medRxiv, 2020, vol. 9, 6 p. DOI: 10.1101/2020.03.23.20041517

13. Kapoor A., Ben X., Liu L., Perozzi B., Barnes M., Blais M., O'Banion S. Examining COVID-19 Forecasting using Spatio-Temporal Graph Neural Networks. arXiv, 2020, vol. 6, pp. 031133.

14. Yesilkanat C.M. Spatio-temporal estimation of the daily cases of COVID-19 in worldwide using random forest machine learning algorithm. Chaos, Solitons and Fractals, 2020, vol. 140, pp. 110210. DOI: 10.1016/j.chaos.2020.110210

15. Jia J.S., Lu X., Yuan Y., Xu G., Jia J., Christakis N.A. Population flow drives spatio-temporal distribution of COVID-19 in China. Nature, 2020, vol. 582, pp. 389-394.

16. Last M. The first wave of COVID-19 in Israel-Initial analysis of publicly available data. PLoS ONE, 2020, vol. 15, no. 10, pp. 1-16. DOI: 10.1371/journal.pone.0240393

17. Bacaer N. A mathematical model of the beginning of the coronavirus epidemic in France. Mathematical Modelling of Natural Phenomena, 2020, vol. 15, pp. 1-10. DOI: 10.1051/mmnp/2020015

18. Coronavirus Cases. Israel. Worldometers. Available at: https://www.worldometers.info/coronavirus/country/israel/ (12.12.2020).

19. Aschwanden C. The false promise of herd immunity. Nature, 2020, vol. 587, no. 7832, pp. 26-28. DOI: $10.1038 / \mathrm{d} 41586-020-02948-4$

20. Yagiz S., Mori J., Miehling E., Basar T., Smith R.L., West M., Mehta P.G. A data-informed approach for analysis, validation, and identification of COVID 19 models. medRxiv, 2020, vol. 6, 8 p. DOI: 10.1101/2020.10.03.20206250

21. Paul S.K., Jana S., Bhaumik P. On nonlinear incidence rate of COVID-19. medRxiv, 2020, vol. 21, 11 p. DOI: $10.1101 / 2020.10 .19 .20215665$

22. Ahmed N., Wei Z., Baleanu D., Rafid M., Rehman M.A. Spatio-temporal numerical modeling of reaction-diffusion measles epidemic system. Chaos: An Interdisciplinary Journal of Nonlinear Science, 2019, vol. 29, no. 10, pp. 103101. DOI: 10.1063/1.5116807

23. Hou X., Gao S., Li Q., Kang Y., Chen N., Chen K., Rao J., Ellenberg J.S., Patz J.A. Intra-county modeling of COVID-19 infection with human mobility: assessing spatial heterogeneity with business traffic, age and race. medRxiv, 2020, vol. 6, 17 p. DOI: 10.1101/2020.10.04.20206/63

24. Polyanin A.D., Vyaz'min A.V. Uravneniya teploprovodnosti i diffuzii s konechnym vremenem relaksatsii. Postanovki zadachi nekotorye resheniya [Heat conduction and diffusion equation with finite relaxation time. Problem definitions and certain solutions]. Izvestiya vysshikh uchebnykh zavedenii. Seriya: Khimiya i khimicheskaya tekhnologiya, 2013, vol. 56, no. 9, pp. 102-108 (in Russian).

25. Sobolev S.L. Transport processes and traveling waves in systems with local nonequilibrium. Soviet Physics Uspekhi, 1991, vol. 3, no. 34, pp. 217-229.

26. Results of the national serological survey for novel coronavirus. Press Release of the Ministry of Health, Israel, 2020. Available at: https://www.gov.il/en/departments/news/08102020-01 (15.11.2020).

Sokolovsky V.L., Furman G.B., Polyanskaya D.A., Furman E.G. Spatio-temporal modeling of COVID-19 epidemic. Health Risk Analysis, 2021, no. 1, pp. 23-37. DOI: 10.21668/health.risk/2021.1.03.eng

Received: 01.02.2021

Accepted: 16.02.2021

Published: 30.03.2021 